\title{
REALIDADE VIRTUAL NA REABILITAÇÃO DE EQUILÍBRIO E MARCHA DO PÓS ACIDENTE VASCULAR CEREBRAL: REVISÃO INTEGRATIVA.
}

\section{Brenda Souza Severo1; Alexssander Weber Crivellaro ${ }^{2}$; Carolina Favero Kerche $^{3}$; Natália Dal Forno ${ }^{4}$ S Sheila Spohr Nedel'; Lucila De Bortoli Da Silva6; Nathaly Marin Hernandez ${ }^{7}$}

\section{RESUMO}

Objetivo: Averiguar os efeitos da realidade virtual em relação ao equilíbrio e marcha em pacientes acometidos pelo Acidente Vascular Cerebral na reabilitação fisioterapêutica. Metodologia: Estudo do tipo revisão integrativa de literatura, foram selecionados artigos das bases de dados PubMed e Scielo, na língua inglesa. Critérios de inclusão: Ensaios clínicos dos últimos cinco anos, com tema sobre o uso da realidade virtual na reabilitação de pacientes pós Acidente Vascular Cerebral e manuscritos gratuitos. Critérios de exclusão: Revisões, estudos incompletos, participação em testes de projetos, desenvolvimento de programas de realidade virtual, outras doenças neurológicas e estudos que citassem outro método como reabilitação principal. Resultado: Foram encontrados 36 artigos, após a leitura mais aprofundada dos resumos e aplicação dos critérios de exclusão restaram apenas 4 artigos. Conclusão: A realidade virtual é um método bastante utilizado na promoção do ganho de amplitude de movimento, equilíbrio e marcha em pacientes participantes dos estudos.

Palavras chaves: Ambiente virtual; Acidente Cerebrovascular; Fisioterapia.

Eixo Temático: Atenção Integral e Promoção à Saúde (AIPS).

\section{INTRODUÇÃO}

Sabe-se que o que o Acidente Vascular Cefálico (AVC) é caracterizado como a $2^{\circ}$ principal patologia crônica não transmissível responsável por mortes no mundo (OMS, 2020). O Ministério da Saúde (2013), descreve o AVC como uma doença de origem vascular de etiologia isquêmica ou hemorrágica, com as respectivas repercussões: interrupção do fluxo sanguíneo cerebral devido a uma obstrução ou

\footnotetext{
${ }^{1}$ Brenda Souza Severo - Universidade Franciscana brenda.s.severo@gmail.com

${ }^{2}$ Alexssander Weber Crivellaro - Universidade Franciscana alexcrivellaro2@gmail.com

${ }^{3}$ Carolina Favero Kerche - Universidade Franciscana carolinakerche99@gmail.com

${ }^{4}$ Natália Dal Forno - Universidade Franciscana natalia.dforno@ufn.edu.br

${ }^{5}$ Sheila Spohr Nedel - Universidade Franciscana sheila.nedel@ufn.edu.br

${ }^{6}$ Lucila De Bortoli Da Silva - Universidade Franciscana lucila.dendrus@gmail.com

${ }^{7}$ Nathaly Marin Hernandez - Universidade Franciscana nathaly.hernandez@ufn.edu.br
} 
rompimento de um vaso que ocasiona uma hemorragia intracerebral.

Siqueira, Schneiders, Silva (2019), citam que a respectiva doença possuí inúmeras variáveis de risco responsáveis por desencadear as repercussões, tais como os não modificáveis (baixo peso ao nascer, idade, sexo, fatores hereditários e etnia) e os modificáveis (diabetes mellitus, tabagismo, síndrome metabólica, álcool e drogas de maneira indiscriminada, hipertensão arterial sistêmica, doenças do coração, dislipidemia e reposição hormonal).

A perda da funcionalidade é a principal consequência em pacientes adultos acometidos pelo AVC gerando um impacto negativo na qualidade de vida e prática de atividades de vida diárias, o que torna essa condição um grave problema de saúde pública (RANGEL; BELASCO; DICCINI, 2013). Como forma de garantir ganhos e recuperação da funcionalidade com boa qualidade, um tratamento com abordagem multiprofissional voltado para a prevenção e mudanças de hábitos diários negativos juntamente com a realização da fisioterapia, torna o processo de reparação positivo, visto que para o indivíduo acometido e o cuidador esse é grande desafio (RAMOS-LIMA et al., 2018; SIQUEIRA; SCHNEIDERS; SILVA, 2019).

A realidade virtual $(R V)$ é uma opção fisioterapêutica utilizada com frequência na reabilitação do pós AVC, através da utilização de mecanismos táteis que permitem a prática de atividades no cenário virtual com base nos movimentos corporais (BARILLI; EBECKEN; CUNHA, 2011; ARAMAKI et al., 2019).

A escolha do tema justifica-se no propósito de investigar as melhoras da função e independência de indivíduos com membros inferiores acometidos pelo AVC com a utilização da RV como método terapêutico, pois acredita-se que ele ofereça diversos benefícios a quem faz o seu uso.

O presente estudo busca responder o seguinte problema de pesquisa: quais os efeitos da RV no equilíbrio e marcha de pacientes pós AVC? O objetivo da pesquisa é averiguar quais são os efeitos da aplicação da RV em relação ao equilíbrio e marcha em pacientes acometidos pelo AVC no período de reabilitação fisioterapêutica. 


\section{QUFN}

\section{METODOLOGIA}

O presente estudo refere-se a uma revisão integrativa de literatura, onde foram selecionados artigos dos últimos cinco anos nas bases de dados PubMed (National Library of Medicine) e Scielo (Scientific Electronic Library Online), com base nos descritores Realidade Virtual, Acidente Vascular Cerebral e Fisioterapia, todos selecionados na língua inglesa e com o uso do operador booleano AND entre os descritores.

Ensaios clínicos dos últimos cinco anos publicados entre 2016 a 2021 na língua inglesa, que possuíssem como tema o uso da RV na reabilitação de pacientes pós AVC com foco na marcha e equilíbrio dos mesmos e apenas manuscritos disponíveis na íntegra de maneira de gratuita foram os critérios de inclusão para o estudo. Os critérios de exclusão foram revisões sistemáticas, estudos incompletos, participação em testes de projetos, desenvolvimento de programas de RV, outras doenças neurológicas e estudos que citassem outro método como reabilitação principal.

\section{RESULTADOS}

Através desta pesquisa, 36 publicações foram encontradas, em que 29 dos resultados pertenceram a base de dados PubMed, onde 25 artigos foram excluídos, restando 4 artigos selecionados. A base de dados Scielo correspondeu a um total de 7 artigos, sendo nenhum se enquadrou nos critérios de inclusão. Um total de 4 artigos foram selecionadosneste estudo.

Figura 1- Fluxograma do processo de exclusão. 
EDUCAÇÃO, SAÚDE

ETECNOLLGIA

26 A 28 DE OUTUBRO DE 2021

\section{QUFN}

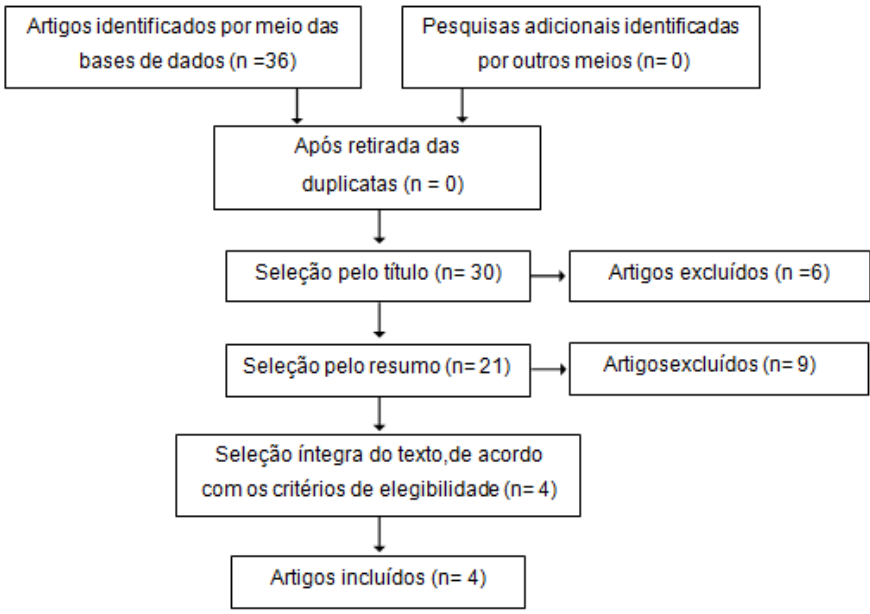

Fonte: autores. 


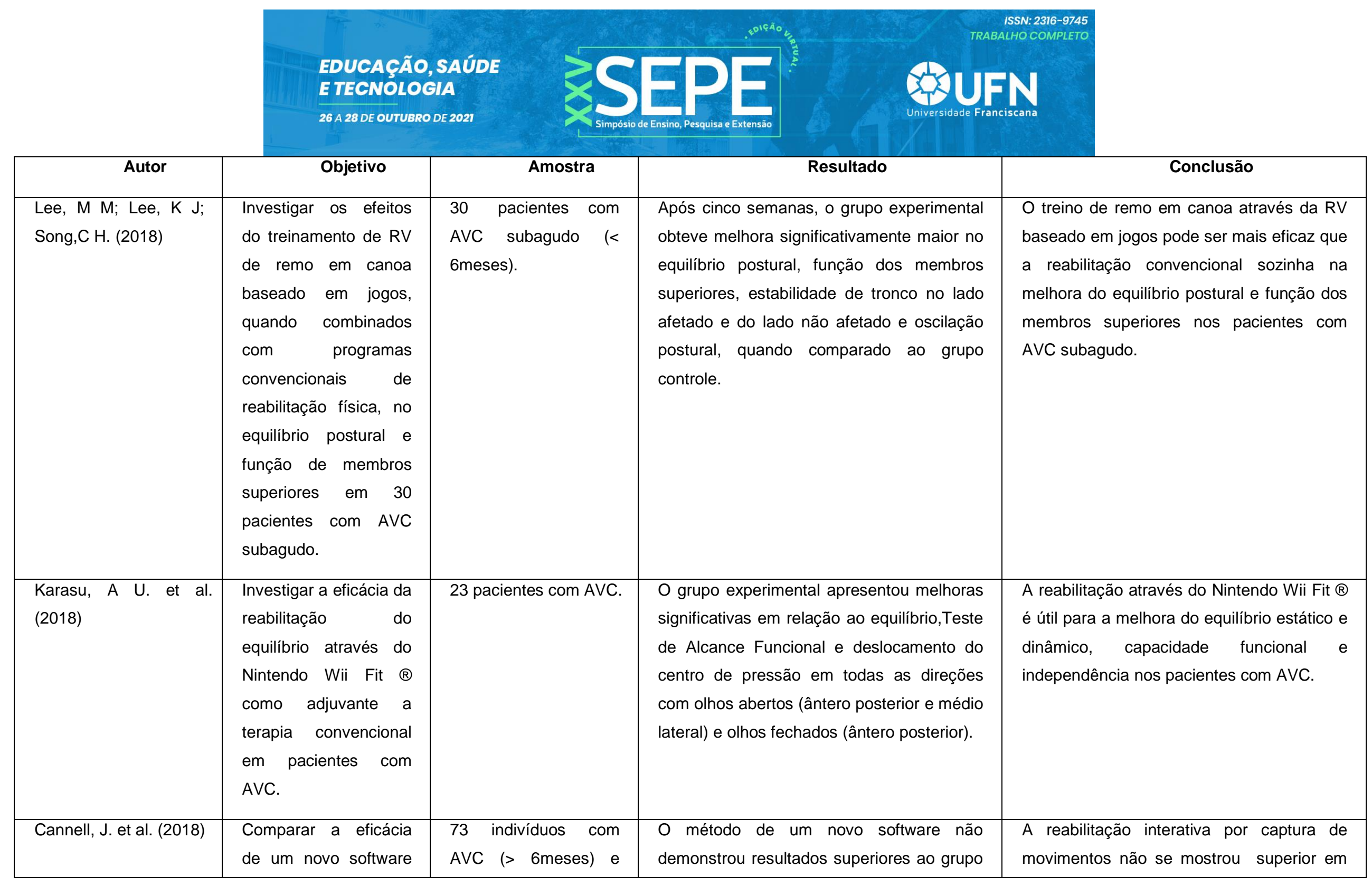




\begin{tabular}{|c|c|c|c|c|}
\hline & $\begin{array}{l}\text { interativo de } \\
\text { reabilitação de captura } \\
\text { de movimento como } \\
\text { tratamento usual na } \\
\text { reabilitação de } \\
\text { pacientes pós AVC na } \\
\text { função física. }\end{array}$ & $\begin{array}{l}\text { com mobilidade } \\
\text { reduzida. }\end{array}$ & controle. & $\begin{array}{l}\text { relação à melhora da função quando } \\
\text { comparado ao tratamento convencional. }\end{array}$ \\
\hline $\begin{array}{l}\text { In,T.; Lee, K.; Song, C. } \\
(2016)\end{array}$ & $\begin{array}{l}\text { Investigar se a terapia } \\
\text { de reflexão de } \mathrm{RV} \\
\text { pode melhorar o } \\
\text { equilíbrio postural e } \\
\text { marcha de pacientes } \\
\text { com AVC crônico. }\end{array}$ & $\begin{array}{l}25 \text { pacientes com AVC } \\
\text { crônico (>6 meses). }\end{array}$ & $\begin{array}{l}\text { Ocorreu melhora significativa no grupo } \\
\text { terapia de reflexão de realidade virtual } \\
(p<0,05) \text { para Escala de Equilíbrio de Berg, } \\
\text { Teste de Alcance Funcional, Time Up And } \\
\text { Go Test, velocidade de caminhada de } 10 \\
\text { metros e oscilação postural, quando } \\
\text { comparados ao grupo controle. }\end{array}$ & $\begin{array}{l}\text { Efeitos benéficos confirmam-se para a } \\
\text { melhora da funcionalidade de membros } \\
\text { inferiores em relação ao equilíbrio e marcha } \\
\text { através da aplicação da terapia de reflexão } \\
\text { de RV nos pacientes com AVC crônico. }\end{array}$ \\
\hline
\end{tabular}

Fonte: pesquisa autores. 


\section{DISCUSSÃO}

Schuster (2014), verificou em seu estudo os efeitos da utilização de Wii reabilitação (uma forma de aplicar a RV) no equilíbrio de pacientes hemiparéticos pós AVC. Ao total de treze pacientes diagnosticados com AVC, seis pacientes foram submetidos a um treinamento funcional com o auxílio do programa interativo do vídeo game Nintendo Wii e os demais realizaram cinesioterapia clássica. Os resultados afirmam que as duas intervenções trazem efeitos positivos e significativos para a melhora do equilíbrio, marcha e funcionalidade dos pacientes hemiparéticos. Sendo assim, o Nintendo Wii é mais um recurso que pode utilizado nos atendimentos de fisioterapia. Karasu et al. (2018), reafirma que a utilização da RV através do Nintendo Wii Fit @ tem resultados positivos também sobre a melhora do equilíbrio estático e dinâmico, capacidade funcional e independência nos pacientes com AVC.

Cannell et al. (2018), realizaram uma comparação entre a eficácia de um novo software interativo de reabilitação de captura de movimento como tratamento usual na reabilitação de pacientes pós AVC na função motora. Mostrou-se nos resultados que o tratamento convencional é superior em relação a melhora da função motora quando comparada ao novo software.

In; Lee ;Song, (2016) apresentam em seu estudo que a terapia de reflexão de $\mathrm{RV}$ tem efeitos satisfatórios em relação a funcionalidade de membros inferiores, equilíbrio e marcha em pacientes pós AVC crônico. No entanto, Bruneo (2021), traz em sua pesquisa de estudos que o treino de RV apresenta "possíveis" melhoras no equilíbrio e marcha dos pacientes quando combinada com a terapia convencional.

Para acrescentar aos achados, Cano-mañas (2017), realizou um estudo prospectivo longitudinal com quatorze pacientes do hospital de La Fuenfría (Espanha), no qual foram avaliados pré e pós-intervenção, utilizando o videoconsole Xbox $360^{\circ}$ (uma forma de aplicar a RV) no tratamento fisioterapêutico que durou oito semanas, obteve-se como resultado que a RV é útil para os pacientes, relacionado a ganho de equilíbrio, controle postural, qualidade de vida, independência nas atividades de vida diária e principalmente em relação a adesão destes participantes à terapia. 
Corroborando com os artigos acima mencionados, Coutinho (2021), realizou uma revisão de literatura, utilizou as bases de dados PubMed, Scopus e Lilacs, para procura dos artigos dos meses de agosto a setembro de 2020, com os descritores "Virtual reality", "Gait" e "Stroke" e com o objetivo de identificar a eficácia e modo de utilização da RV na reabilitação da marcha pós-AVC. Ele conclui através deste estudo que a utilização da RV na reabilitação da marcha após AVC pode ser utilizada de diferentes formas e traz resultados positivos no tratamento dos pacientes, sendo um recurso de simples aplicabilidade, acessível e de fácil utilização. A RV também se mostra efetiva para a melhora da funcionalidade, mobilidade, equilíbrio, tônus, além de oferecer motivação durante o tratamento dos pacientes pós AVC.

Lee; Lee; Song (2018), apresentaram em seu estudo que o treino de remo em canoa através da RV baseado em jogos pode apresentar resultados mais positivos na melhora do equilíbrio postural e função de membros superiores quando comparado a fisioterapia convencional sozinha aoser mais eficaz que a reabilitação convencional sozinha. Marciano et al. (2021), reforça que a utilização da RV traz resultados mais positivos no tratamento de pacientes pós AVC em comparação com a terapia convencional. Desta forma, a RV é uma conduta complementar ao tratamento fisioterapêutico e os resultados trazem a importância da utilização dessa intervenção na prática clínica para o tratamento de pacientes pós AVC.

\section{CONCLUSÃO:}

A RV é um método seguro praticável na reabilitação de pacientes de várias patologias, dentre elas pacientes pós AVC, pois se trata de um tratamento alternativo e eficaz utilizado durante os atendimentos fisioterapêuticos.

Segundo os autores mencionados é possível afirmar que método da RV contribuiu na melhora da funcionalidade de membros inferiores, equilíbrio e marcha das amostras encontradas nos estudos.

Com isso, pode-se concluir que a RV é uma alternativa que pode ser cada vez mais utilizada na reabilitação dos pacientes pós AVC em relação ao equilíbrio e marcha, sendo esta uma opção mais interativa e motivadora. 


\section{REFERÊNCIAS BIBLIOGRÁFICAS}

ARAMAKI, Alberto Luiz et al. Virtual reality in the rehabilitation of patients with stroke: an integrative review. Arquivos de neuro-psiquiatria, v.77, n.4, p.268-278, 2019.

BARILLI, Elomar Christina Vieira Castilho; EBECKEN, Nelson Francisco Favilla; CUNHA, Gerson Gomes. A tecnologia de realidade virtual como recurso para formação em saúde pública à distância: uma aplicação para a aprendizagem dos procedimentos antropométricos. Ciência \& Saúde Coletiva, v.16, p.1247-1256, 2011.

Brasil. Ministério da Saúde. Secretaria de Atenção à Saúde. Departamento de Ações Programáticas Estratégicas. Diretrizes de atenção à reabilitação da pessoa com acidentevascular cerebral/Ministério da Saúde, Secretaria de Atenção à Saúde, Departamento de Ações Programáticas Estratégicas -Brasília: Ministério da Saúde, 2013.

BRUNEO, Filomeri. $O$ efeito realidade virtual na reabilitação da marcha e equilíbrio em pacientes após AVE fase crónica: uma revisão bibliográfica. 2021. Trabalho de Conclusão de Curso (TCC), Faculdade de Fisioterapia, Escola Superior de Saúde Fernando Pessoa, 2021.

CANNELL, John et al. The efficacy of interactive, motion capture-based rehabilitation on function al outcomes in an in patient stroke population: a randomized controlled trial. Clinical rehabilitation, v. 32,n. 2, p.191-200, 2018.

CANO-MAÑAS, María J.; COLLADO-VÁZQUEZ, Susana; CANO-DE-LA-CUERDA, Roberto. Videojuegos comerciales en la rehabilitación de pacientes con ictus subagudo: estudio piloto. Revista de Neurologia, v. 65, n. 8, p. 337-347, 2017.

MARCIANO, Ingrid Alcântara et al. Efeitos da intervenção fisioterapêutica associada à realidade virtual na reabilitação de pacientes pós-AVE. 2021.

COUTINHO, Dinara Laiana de Lima Nascimento. Realidade virtual na reabilitação da marcha após acidente vascular cerebral: Uma revisão de literatura. Revista SaúdeUNG-Ser, v. 15, n. 1/2, p. 62-69, 2021.

IN, Taesung; LEE, Kyeongjin; SONG, Changho. Virtual reality reflection therapy improves balance and gait in patients with chronic stroke: randomized controlled 
trials. Medical science monitor: international medical journal of experimental and clinical research, v.22, p.4046, 2016.

KARASU, Ayça Utkan et al. Effectiveness of Wii-based rehabilitation in stroke: a randomized controlled study. Journal of rehabilitation medicine, v. 50, n. 5, p. 406412, 2018.

LEE, Myung Mo; LEE, Kyeong Jin; SONG, Chang Ho. Game-based virtual reality canoe paddling training to improve postural balance and upper extremity function: A preliminary randomized controlled study of 30 patients with subacute stroke. Medical science monitor: international medical journal of experimental and clinical research, v. 24, p.2590, 2018.

RAMOS-LIMA, Maria José Melo et al. Quality of life after stroke: impact of clinical and socio demographic factors. Clinics, v. 73, 2018.

RANGEL, Edja Solange Souza; BELASCO, Angélica Gonçalves Silva; DICCINI, Solange. Qualidade de vida de pacientes com acidente vascular cerebral em reabilitação. Acta paulista de enfermagem, v. 26,n.2,p.205-212, 2013.

SCHUSTER, Rodrigo Costa. Efeitos da realidade virtual no equilíbrio de indivíduos hemiparéticos. Fisioterapia Brasil, v. 15, n. 1, p. 49-55, 2014.

SIQUEIRA, Sandro; DE BORBA SCHNEIDERS, Paloma; GONÇALVES DA SILVA, Andréa Lúcia. Intervenções fisioterapêuticas e sua efetividade na reabilitação do paciente acometido por acidente vascular cerebral. Fisioterapia Brasil, v. 20, n. 4, 2019.

World Heatlh Organization. (WHO). The top 10 causes of death.Genebra; 2020. Available from: https://www.who.int/news-room/fact-sheets/detail/the-top-10causes-of-death. Acesso em 08/05/2021 as 15:21. 